\title{
Die Lohnentwicklung in Deutschland zwischen 1998 und 2005 - Wachsende Ungleichheit
}

\author{
Johannes Giesecke \\ Roland Verwiebe
}

\begin{abstract}
Die Lohnungleichheit in Deutschland hat sich seit Ende der 1990er Jahre deutlich verstärkt. Außerdem zeigt sich, dass die wachsenden Lohndifferenzen sozial strukturiert sind: Insbesondere untere Berufsklassen verzeichneten relative Lohnverluste. Darüber hinaus trägt die Ausweitung von Dienstleistungs- und Teilzeitjobs zu einer Spreizung der Löhne am deutschen Arbeitsmarkt bei.
\end{abstract}

\section{Aktuelle Entwicklung der Lohn- und Einkommensungleichheit}

Die Bundesrepublik wies im internationalen Vergleich über viele Jahrzehnte ein im Durchschnitt deutlich geringeres Ausmaß der ungleichen Verteilung von Löhnen und Einkommen auf als die meisten anderen entwickelten kapitalistischen Gesellschaften (Atkinson 2000). Zudem waren die Ungleichheitsrelationen in Deutschland im Zeitverlauf durch eine hohe Stabilität gekennzeichnet (Katz/Autor 1999; Prasad 2004; Steiner/Hölzle 2000), d.h. die Relationen zwischen unterschiedlichen sozialen Gruppen der Gesellschaft blieben über viele Jahre mehr oder weniger unverändert. Diese relative Stabilität der Ungleichheitsstrukturen ist insofern bemerkenswert, da in vielen anderen Nationen zwischen Anfang der 1980er und Mitte der 1990er Jahre - genannt werden können z. B. Italien, Großbritannien, die USA, Japan, Schweden oder Neuseeland (Katz/Autor 1999, S. 1503) - ein zum Teil deutlicher Anstieg der ungleichen Verteilung von Einkommen und Löhnen beobachtet werden konnte. Allerdings legen neuere Forschungsergebnisse für den Zeitraum ab Ende der 1990er Jahre nahe, dass die ungleiche Verteilung von Ressourcen und Lebenschancen auch in der Bundesrepublik wieder zugenommen hat (Berger 2005; Bergmann 2004; Gernandt/Pfeiffer 2006; Hradil 2005; Kohn 2006; Möller 2005; Schettkat 2006).

Schon eine Betrachtung allgemeiner Kennziffern belegt die Relevanz des angesprochenen Phänomens. Mit Blick auf die Lohnentwicklung seit Mitte der 1990er Jahre ist die Bundesrepublik unter den westlichen Industrienationen das Land mit der negativsten Entwicklung der Arbeitslöhne. ${ }^{1}$ Dieser Entwicklung der Einkommen aus nicht-selbstständiger Arbeit steht die Entwicklung der Kapitaleinkommen entgegen: Deutsche Aktiengesellschaften schütteten 2005 knapp 30 Mrd. $€$ Dividenden an Kapitaleigner aus, ein historischer Rekord (DSW 2006a). Die Einkommensschere zwischen Arbeitnehmerentgelt und Unternehmenseinkommen hat sich daher weiter geöffnet (Schäfer 2006; Statistisches Bundesamt 2007). Das skizzierte Bild lässt sich abrunden durch die Entwicklung der Jahresgehälter der Vorstandsmitglieder der DAX-30-Unternehmen, die sich zwischen 1997 und 2005 (ohne Bonuszahlungen) von 1,66 Mio. DM auf 1,7 Mio. $€$ verdoppelten (DSW 2006b).

Wenn also gesamtgesellschaftliche Ungleichheit in der Bundesrepublik wieder zunimmt, dann ist die Frage soziologisch relevant, wie unterschiedliche soziale Gruppen von dieser Entwicklung konkret betroffen sind. Zuspitzen kann man dies auf die Frage, wer die Gewinner und wer die Verlierer einer veränderten Verteilung von gesellschaftlichem Wohlstand sind. Diesem Problem widmet sich der vorliegende Beitrag. Dabei liegt der Fokus auf der Betrachtung individueller Brutto-Arbeitslöhne abhängig Beschäftigter und der damit identifizierbaren Ungleichheitsstrukturen. Empirisch wird dazu in zwei Schritten vorgegangen. Im Anschluss an eine kurze Skizze unserer Forschungsmethodik (Abschnitt 2) werden zunächst die deskriptiven Trendergebnisse für den Zeitraum von 1998 bis 2005 vorgestellt (Abschnitt 3.1) womit wir direkt an bereits vorliegende Analysen, die den Zeitraum von 1990 bis 1997 ausgewertet haben, anknüpfen (Prasad 2004; Steiner/Hölzle 2000). In einem zweiten Schritt wird mithilfe von Dekompositionsmethoden der Frage nachgegangen, ob die wachsende Lohnungleichheit in Deutschland auf eine veränderte Wirkungsweise ungleichheitsgenerierender Mechanismen und/oder auf eine veränderte soziale Zusammensetzung der Erwerbstätigen zurückführbar ist (Abschnitt 3.2). Als ungleichheitsgenerierende Mechanismen bezeichnen wir Differenzen in den (mittleren) Löhnen von Gruppen am Arbeitsmarkt, wie z. B. Lohnunterschiede zwischen Hoch- und Niedrigqualifizierten oder zwischen deutschen und nicht-deutschen Arbeitnehmerinnen und Arbeitnehmern. Diese Lohndifferenzen können im Zeitverlauf größer oder kleiner werden, was sich etwa in einer wachsenden oder sinkenden Lohndiskriminierung von Ausländern zeigen würde. Aber auch die $\mathrm{Zu}$ sammensetzung der Erwerbstätigen nach bestimmten Merkmalen bzw. ihre Veränderung kann Konsequenzen für die ungleiche Verteilung der Löhne haben. So könnte beispielsweise eine Expansion von Jobs im Dienstleistungssektor zu einer Verstär-

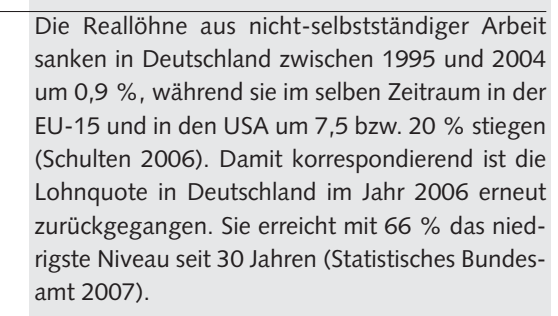
sanken in Deutschland zwischen 1995 und 2004 um $0,9 \%$, während sie im selben Zeitraum in der EU-15 und in den USA um 7,5 bzw. $20 \%$ stiegen (Schulten 2006). Damit korrespondierend ist die Lohnquote in Deutschland im Jahr 2006 erneut zurückgegangen. Sie erreicht mit $66 \%$ das niedrigste Niveau seit 30 Jahren (Statistisches Bundesamt 2007)

Johannes Giesecke, Dr., Wissenschaftszentrum Berlin für Sozialforschung. Arbeitsschwerpunkte: Arbeitsmarkt, Ungleichheit, quantitative Methoden. e-mail: giesecke@wzb.eu Roland Verwiebe, Dr., Universität Hamburg, Institut für Soziologie, Methoden der empirischen Sozialforschung. Arbeitsschwerpunkte: Ungleichheit, Migration, Mobilität. e-mail: roland.verwiebe@uni-hamburg.de 
kung der Lohnspreizung führen, wenn Tätigkeiten im Dienstleistungssektor nur unterdurchschnittlich entlohnt werden. Diese Unterscheidung orientiert sich an der ökonomischen Literatur, in der eine veränderte Lohnverteilung auf eine Veränderung der Preise (ungleichheitsgenerierende Mechanismen) und/oder der Quantitäten (Zusammensetzung der Gruppe der Erwerbstätigen) zurückgeführt wird (Juhn et al. 1993). Die getroffene konzeptionelle Unterscheidung zwischen ungleichheitsgenerierenden Mechanismen und sozialer Zusammensetzung der Arbeitnehmerschaft ist aus arbeitsmarktsoziologischer Sicht relevant: Während die Mechanismen den ungleichen Prozess der Entlohnung individueller oder struktureller Merkmale abbilden, ist die soziale Zusammensetzung der Erwerbstätigen ein Resultat ungleicher Zuweisungsprozesse von Personen auf Positionen. $^{2}$

\section{Forschungsmethodik}

\subsection{DATEN, KRITERIEN DER FALLAUSWAHL}

Für die Analysen dieses Beitrags wurden Daten des Sozio-ökonomischen Panels (SOEP) aus den Erhebungsjahren 1998 bis 2005 verwendet (SOEP Group 2001). Die Auswahl der Welle 1998 als Startpunkt des Zeitvergleichs erfolgte neben inhaltlichen Überlegungen (Anschlussfähigkeit an bereits bestehende Analysen) auch aufgrund der in diesem Jahr neu erhobenen Ergänzungsstichprobe, die die zu analysierende Fallzahl deutlich erhöht. ${ }^{3}$

Die empirischen Analysen beziehen sich ausschließlich auf abhängig Beschäftigte im erwerbsfähigen Alter ( 16 bis 65 Jahre), die zum Befragungszeitpunkt weder arbeitslos gemeldet noch in einer Arbeitsbeschaffungsmaßnahme bzw. in einem Ausbildungsverhältnis beschäftigt waren. In den Modellen wurden Beobachtungen derjenigen Personen mit Bruttostundenlöhnen von weniger als $2,5 €$ (in konstanten Preisen von 1998) ausgeschlossen, um Effekte von Ausreißern möglichst niedrig zu halten.

Die Analysen wurden für West- und Ostdeutschland sowie für Männer und Frauen getrennt berechnet. Insgesamt konnten für 1998 (2005) Informationen von westdeutschen 2.381 (2.755) Männern und 1.689 (2.370) Frauen, 802 (791) ostdeutschen Männern und 713 (788) Frauen genutzt werden.

\subsection{VARIABLEN, METHODEN}

Die für die Analysen zentrale Variable Bruttostundenlohn ergibt sich aus dem Verhältnis des erzielten monatlichen Bruttoarbeitseinkommens (in $€$ ) und der tatsächlich geleisteten Arbeitszeit (inklusive Überstunden). Um die Angaben über die verschiedenen Messzeitpunkte vergleichen zu können, wurden die Löhne durch den Einbezug jährlicher Inflationsraten auf konstante Preise von 1998 umgerechnet. ${ }^{4}$

Als Variablen, die in den Dekompositionsmodellen für die Erklärung des Wandels der Lohnungleichheit in Deutschland zwischen 1998 und 2005 genutzt werden, fungieren: Alter, Bildungsniveau, Dauer der Betriebszugehörigkeit, Arbeitslosigkeit in den letzten zwölf Monaten, Nationalität, Vertragsart (Befristung), Arbeitszeit, Branche, Betriebsgröße, berufliche Position (Klassenposition $)^{5}$ sowie Anzahl der Kinder im Haushalt und Familienstatus.

Die Dekompositionsanalyse beruht auf der von Juhn et al. (1993) vorgeschlagenen Methode, mit deren Hilfe die Änderung der Lohnungleichheit zwischen zwei Zeitpunkten in insgesamt drei Komponenten zerlegt werden kann: eine veränderte Wirkungsweise ungleichheitsgenerierender Mechanismen, eine veränderte Zusammensetzung der Gruppe der Erwerbstätigen und eine veränderte Wirkung unbeobachteter Einflussfaktoren. Formal gesehen liegen dieser Zerlegung mehrere Regressionsanalysen zugrunde, die allerdings aus Platzgründen hier nicht dargestellt werden können. ${ }^{6}$ In Ergänzung zu diesem Ansatz wird hier der Versuch unternommen, in detaillierten Analysen den Beitrag einzelner, individueller bzw. arbeitsplatzbezogener Merkmale (wie Bildung oder Klassenposition) zu untersuchen.

\section{Anstieg der Lohnungleichheit}

Im ersten Teil dieses Abschnitts wird zunächst deskriptiv die Entwicklung der Verteilung der Arbeitslöhne und die damit einhergehende Veränderung der Lohnungleichheit im Zeitraum von 1998 bis 2005 diskutiert. Da es sich hierbei um Aggregatdaten handelt, die nur bedingt einen Rückschluss auf zugrunde liegende sozialstrukturelle Dynamiken gestatten, wird im zweiten Teil mit der Diskussion der Ergebnisse der Lohndekomposition die Zunahme von Lohnungleichheit noch detaillierter untersucht.

\subsection{DESKRIPTIVE ANALYSEN}

Die Entwicklung der Bruttolöhne aus nicht-selbstständiger Arbeit zwischen 1998 und 2005 lässt sich zunächst gut mit den in Abbildung 1 dargestellten Lohndezilen [erstes (D1), fünftes (D5) und neuntes (D9) Dezil] diskutieren. Dabei ist zu erkennen, dass zwischen Männern und Frauen, aber auch zwischen den alten und den neuen Bundesländern, erhebliche Unterschiede in der Lohnverteilung und deren zeitlicher Entwicklung existieren. Fünf Punkte sollen hier hervorgehoben werden.

Erstens lässt sich für Männer in den alten Bundesländern für den Zeitraum 1998 bis 2005 ein Anstieg der oberen und mittleren Löhne bei gleichzeitigem Absinken der unteren Löhne beobachten. ${ }^{7}$ Zugleich wird deutlich, dass die absoluten Abstände zwischen den betrachteten Lohnbereichen bei Männern aus Westdeutschland insgesamt am prägnantesten ausgeprägt sind. Zweitens ist für Männer in Ostdeutschland die stärkste Zunahme der Lohnabstände innerhalb des Beobachtungszeitraums fest-

2 Empirisch treten beide Phänomene vermutlich fast immer gleichzeitig auf, wobei sie sich jedoch in ihrer Bedeutung für die Erklärung einer sich verändernden Lohnverteilung unterscheiden können.

3 Die Stichprobe G (Hocheinkommensbezieher) konnte aufgrund der fehlenden Gewichtungsinformationen nicht genutzt werden.

4 Die dafür notwendigen Informationen sind der elektronischen Datenbank „International Financial Statistics" entnommen.

5 Hierfür wurde die Klassifikation von Erikson und Goldthorpe (1992) verwendet.

6 Die Ergebnisse sind jedoch auf Nachfrage von den Autoren erhältlich.

7 Der Lohn auf dem D9-Niveau stieg von 22,5 auf $24,0 €$, der auf dem D1-Niveau sank von 9,0 auf $8,80 €$. Das D9-Niveau repräsentiert hierbei denjenigen Lohn, der das oberste Zehntel von den übrigen $90 \%$ der Lohnempfänger abtrennt (d.h. $10 \%$ verdienen einen Lohn oberhalb, $90 \%$ einen Lohn unterhalb dieses Lohns). Umgekehrt markiert der Lohn auf dem D1-Niveau die Trennlinie zwischen dem untersten Zehntel der Lohnempfänger und den übrigen $90 \%$. 


\section{Abb. 1: Lohnverteilung von Frauen und Männern zwischen 1998 und 2005 - in Euro, inflationsbereinigt -}
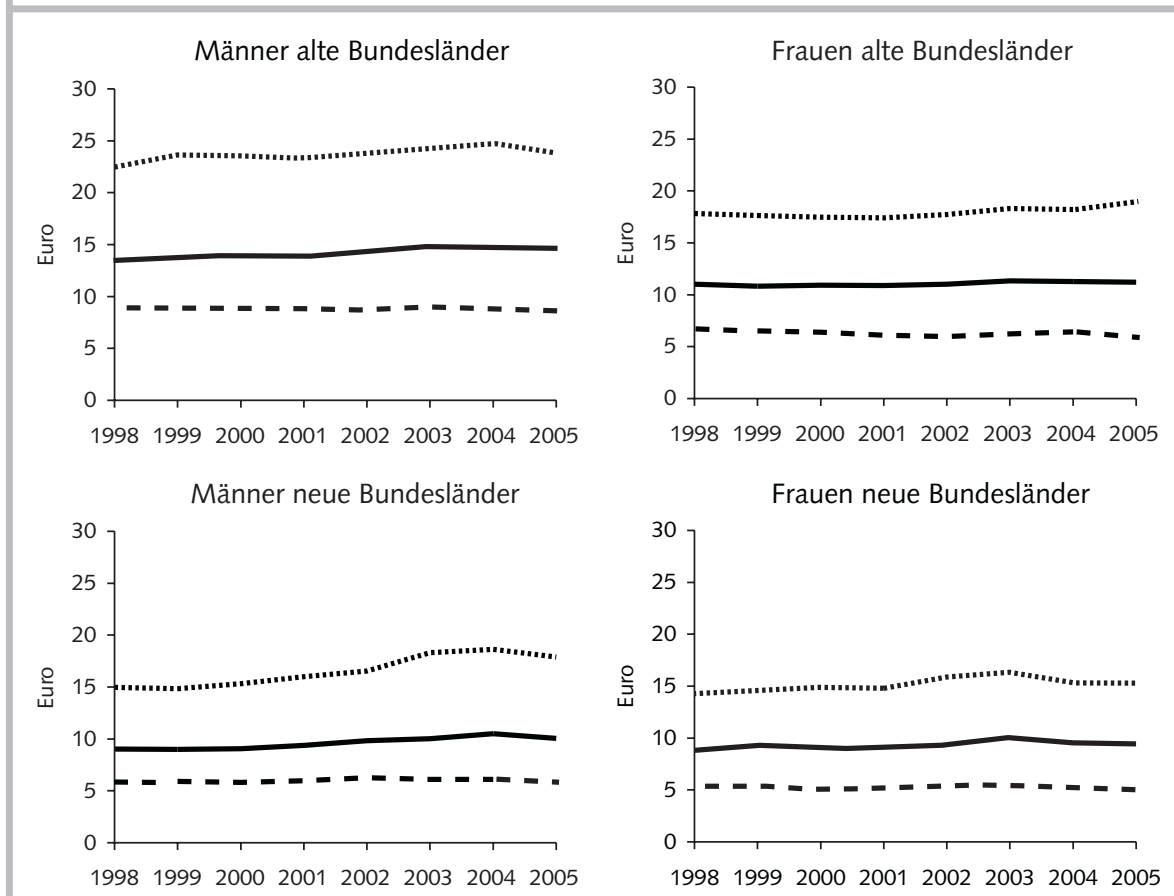

$$
\text { …… D9 }-\mathrm{D} 5 \text { - - - D1 }
$$

Quelle: SOEP 1998-2005; D1: erstes, D5: fünftes, D9: neuntes Dezil; querschnittgewichtet; Berechnungen und Abbildung der Autoren.

\section{WSI MITTELLUNGEN}

zustellen. Diese Einkommensdynamik wurde in erster Linie von einem Anstieg der oberen und mittleren Löhne getragen. ${ }^{8}$ Dennoch fielen auch noch im Jahre 2005 die Lohnabstände innerhalb dieser Gruppe insgesamt geringer aus als für Männer in Westdeutschland. Drittens wird deutlich, dass auch bei Arbeitnehmerinnen in Westdeutschland vor allem die oberen, aber auch die mittleren Reallöhne zwischen 1998 und 2005 gestiegen sind. Dagegen entwickelten sich deren Löhne im unteren Einkommensbereich rückläufig. Insgesamt erweisen sich die Abstände der Reallöhne

bei Frauen aus den alten Bundesländern als relativ stark ausgeprägt. Viertens lässt sich für Frauen aus den neuen Bundesländern im Zeitverlauf eine ähnliche Dynamik wie bei den Frauen in Westdeutschland feststellen: Die Löhne vor allem im oberen, aber auch im mittleren Bereich stiegen, während die unteren Reallöhne stagnierten. Fünftens schließlich wird mit Blick auf die Lohnunterschiede zwischen Männern und Frauen bestätigt, dass Frauen sehr häufig geringere Löhne als Männer erzielen (Hinz/Abraham 2005; Liebeskind 2004; Weins 2005). Dies trifft für alle hier be-

\section{Tabelle 1: Lohnungleichheit zwischen 1998 und 2005} (Dezilverhältnisse)

\begin{tabular}{|c|c|c|c|c|c|c|c|c|c|c|c|c|}
\hline \multirow[b]{3}{*}{ Jahr } & \multicolumn{6}{|c|}{ Alte Bundesländer } & \multicolumn{6}{|c|}{ Neue Bundesländer } \\
\hline & \multicolumn{3}{|c|}{ Männer } & \multicolumn{3}{|c|}{ Frauen } & \multicolumn{3}{|c|}{ Männer } & \multicolumn{3}{|c|}{ Frauen } \\
\hline & $\mathrm{D} 9 / \mathrm{D} 1$ & $\mathrm{D} 9 / \mathrm{D} 5$ & $\mathrm{D} 5 / \mathrm{D} 1$ & $\mathrm{D} 9 / \mathrm{D} 1$ & $\mathrm{D} 9 / \mathrm{D} 5$ & $\mathrm{D} 5 / \mathrm{D} 1$ & $\mathrm{D} 9 / \mathrm{D} 1$ & $\mathrm{D} 9 / \mathrm{D} 5$ & $\mathrm{D} 5 / \mathrm{D} 1$ & $\mathrm{D} 9 / \mathrm{D} 1$ & $\mathrm{D} 9 / \mathrm{D} 5$ & $\mathrm{D} 5 / \mathrm{D} 1$ \\
\hline 1998 & 2,50 & 1,66 & 1,51 & 2,65 & 1,62 & 1,64 & 2,58 & 1,65 & 1,56 & 2,73 & 1,60 & 1,70 \\
\hline 1999 & 2,62 & 1,68 & 1,56 & 2,59 & 1,59 & 1,62 & 2,51 & 1,63 & 1,54 & 2,67 & 1,54 & 1,74 \\
\hline 2000 & 2,64 & 1,69 & 1,57 & 2,65 & 1,58 & 1,68 & 2,63 & 1,69 & 1,56 & 2,95 & 1,65 & 1,78 \\
\hline 2001 & 2,60 & 1,67 & 1,56 & 2,75 & 1,57 & 1,75 & 2,59 & 1,70 & 1,53 & 2,86 & 1,64 & 1,75 \\
\hline 2002 & 2,70 & 1,65 & 1,63 & 2,82 & 1,62 & 1,75 & 2,62 & 1,67 & 1,57 & 3,02 & 1,71 & 1,77 \\
\hline 2003 & 2,64 & 1,63 & 1,62 & 2,94 & 1,62 & 1,82 & 2,93 & 1,80 & 1,62 & 3,00 & 1,63 & 1,84 \\
\hline 2004 & 2,73 & 1,67 & 1,63 & 2,76 & 1,60 & 1,72 & 3,05 & 1,74 & 1,76 & 2,93 & 1,61 & 1,82 \\
\hline 2005 & 2,71 & 1,63 & 1,66 & 3,01 & 1,65 & 1,82 & 3,04 & 1,76 & 1,73 & 2,95 & 1,60 & 1,85 \\
\hline
\end{tabular}

trachteten Bereiche der Lohnverteilung (D1, D5 und D9) zu. Ebenso zeigt sich, dass diese Lohnabstände in den alten Bundesländern wesentlich stärker ausgeprägt sind als in den neuen Bundesländern (Holst 2003). Gleichzeitig lassen die Daten erkennen, dass die Lohnabstände zwischen Männern und Frauen in den neuen Bundesländern während der letzten Jahre zugenommen haben. Noch zu Beginn des Untersuchungszeitraums lagen beispielsweise die oberen Löhne für Männer und Frauen enger beieinander als dies im Jahre 2005 der Fall war, was auf einen sehr starken Anstieg der Reallöhne bei den Männern in diesem Bereich zurückzuführen ist. ${ }^{9}$

Die dargestellten Befunde eignen sich gut für eine allgemeine Charakterisierung der Lohndynamik in Deutschland. Was sich aus der obigen Grafik jedoch nicht ohne weiteres ablesen lässt, ist das Ausmaß der Lohnungleichheit, die in der Regel als relational definiert wird. Daher sollen im Folgenden die Verhältnisse der oben diskutierten Dezile betrachtet werden, mit denen Veränderungen der Ungleichheit im oberen und unteren Bereich der Lohnverteilung dargestellt werden können (Tabelle 1). ${ }^{10}$ Für die entsprechenden Berechungen sind vier Punkte relevant:

Erstens lässt sich für Männer aus den alten Bundesländern bei der D9/D1-Relation, die ein gutes Maß für die Gesamtungleichheit eines Lohngefüges darstellt, ein Anstieg innerhalb des Beobachtungszeitraums erkennen (Anstieg von 2,50 auf 2,71). ${ }^{11}$ Diese Zunahme ist jedoch ausschließlich auf eine verstärkte Spreizung im unteren Bereich der Lohnverteilung zu-

8 Der Zuwachs betrug für das D9-Niveau 3,1 $€$ und $1,1 €$ für das Medianeinkommen (D5).

91998 lag der D9-Lohn bei 14,30 € für Frauen und $15,0 €$ für Männer. Im Jahre 2005 betrug er hingegen $15,50 €$ für Frauen und $18,10 €$ für Männer.

10 Zur Charakterisierung des Ausmaßes der Lohnungleichheit werden in empirischen Studien unterschiedliche Maße verwendet. Neben Dezil-Relationen sind Gini- oder Theil-Indizes gebräuchlich, die jedoch den Nachteil haben, dass sie aufgrund ihrer Konstruktion nur die Gesamtungleichheit einer Verteilung charakterisieren.

11 Eine Veränderung der D9/D1-Relation von 2,50 auf 2,71 kann wie folgt gelesen werden: Der oberste Lohn im neunten Dezil war 1998 um den Faktor 2,50 oder um 150 \% höher als der oberste Lohn im ersten Dezil. Im Jahr 2005 ist die Lohnungleichheit gestiegen: Der oberste Lohn im neunten Dezil ist um 171 \% höher als der oberste Lohn im ersten Dezil. 
rückzuführen (D5/D1-Relation). Gleichzeitig hat sich die Spreizung im oberen Bereich der Lohnverteilung leicht verringert. Zweitens zeigt sich, dass die Ungleichheit der realen Löhne bei Arbeitnehmerinnen in den alten Ländern im selben Zeitraum ebenfalls zugenommen hat. Jedoch fällt die Zunahme der Ungleichheit stärker aus als bei den Männern, was den Befunden von Steiner und Hölzle (2000) zur Entwicklung der Lohnungleichheit in den 1990er Jahren entgegenläuft. Darüber hinaus lässt sich erkennen, dass die Zunahme der Lohnungleichheit vor allem auf Veränderungen in der unteren Hälfte der Lohnverteilung zurückgeht. Drittens wird mit Blick auf die Männer in Ostdeutschland deutlich, dass die auch hier zu konstatierende Zunahme der Ungleichheit insbesondere auf die verstärkte Spreizung der Löhne im unteren Lohnbereich zurückzuführen ist (D5/D1 von 1,56 auf 1,73). Die Ungleichheit im oberen Lohnbereich (D9/D5-Relation) hat im Beobachtungszeitraum zwar auch zugenommen, jedoch fiel der Zuwachs der Ungleichheit im unteren Bereich der Lohnverteilung wesentlich stärker aus. Insgesamt führte die vergleichsweise starke Zunahme der Lohnspreizung bei den Männern in den neuen Bundesländern dazu, dass zum Ende des Beobachtungszeitraums die Gesamtungleichheit über dem Niveau bei den Männern in Westdeutschland lag (Kohn 2006; Möller 2005). Viertens schließlich weisen die Daten darauf hin, dass sich die Lohnungleichheit auch bei Frauen in den neuen Bundesländern erhöht hat (Anstieg von 2,73 auf 2,95). Dieser Zunahme liegt hauptsächlich eine verstärkte Spreizung der unteren Löhne (D5/D1-Relation) zugrunde. Die Ungleichheit im oberen Bereich der Lohnverteilung hat sich hingegen nicht verändert. Darüber hinaus zeigt sich, dass trotz der zunehmenden Ungleichheit der Löhne das Ausmaß der Lohnspreizung für Frauen in den neuen Bundesländern geringer ausfällt als bei Frauen in den alten Bundesländern. Damit geht einher, dass auch der Unterschied in der Lohnungleichheit zwischen Männern und Frauen in Ostdeutschland insgesamt weniger stark ausgeprägt ist als in Westdeutschland.

Insgesamt lassen die Ergebnisse der deskriptiven Analysen der Lohnverteilung den Schluss zu, dass es zu einer deutlichen Zunahme der Ungleichheit der Lohnverteilung sowohl in West- als auch in Ostdeutschland zwischen 1998 und 2005 kam.

Tabelle 2: Dekomposition der Ungleichheitsrelationen für die alten und neuen Bundesländern (Veränderung 1998-2005)

\begin{tabular}{|c|c|c|c|c|}
\hline & $\begin{array}{l}\text { Gesamt- } \\
\text { änderung }\end{array}$ & $\begin{array}{c}\text { Mechanismen } \\
\text { Ungleichheit }\end{array}$ & $\begin{array}{c}\text { Zusammensetzung } \\
\text { Erwerbstätige }\end{array}$ & Unbeobachtet \\
\hline \multicolumn{5}{|l|}{ Westdeutschland } \\
\hline \multicolumn{5}{|l|}{ Männer } \\
\hline $\ln (\mathrm{d} 9 / \mathrm{d} 1)_{2005}-\ln (\mathrm{d} 9 / \mathrm{d} 1)_{1998}$ & 0.082 & 0.089 & -0.041 & 0.034 \\
\hline $\ln (\mathrm{d} 9 / \mathrm{d} 5)_{2005}-\ln (\mathrm{d} 9 / \mathrm{d} 5)_{1998}$ & -0.019 & 0.024 & -0.055 & 0.013 \\
\hline $\ln (\mathrm{d} 5 / \mathrm{d} 1)_{2005}-\ln \left(\mathrm{d} 5 / \mathrm{d}_{1}\right)_{1998}$ & 0.100 & 0.064 & 0.015 & 0.021 \\
\hline \multicolumn{5}{|l|}{ Frauen } \\
\hline $\ln (\mathrm{d} 9 / \mathrm{d} 1)_{2005}-\ln (\mathrm{d} 9 / \mathrm{d} 1)_{1998}$ & 0.128 & 0.098 & 0.018 & 0.011 \\
\hline $\ln (\mathrm{d} 9 / \mathrm{d} 5)_{2005}-\ln (\mathrm{d} 9 / \mathrm{d} 5)_{1998}$ & 0.022 & 0.050 & -0.027 & -0.000 \\
\hline $\ln (\mathrm{d} 5 / \mathrm{d} 1)_{2005}-\ln (\mathrm{d} 5 / \mathrm{d} 1)_{1998}$ & 0.106 & 0.048 & 0.046 & 0.012 \\
\hline \multicolumn{5}{|l|}{ Ostdeutschland } \\
\hline \multicolumn{5}{|l|}{ Männer } \\
\hline $\ln (\mathrm{d} 9 / \mathrm{d} 1)_{2005}-\ln (\mathrm{d} 9 / \mathrm{d} 1)_{1998}$ & 0.166 & 0.126 & 0.018 & 0.021 \\
\hline $\ln (\mathrm{d} 9 / \mathrm{d} 5)_{2005}-\ln (\mathrm{d} 9 / \mathrm{d} 5)_{1998}$ & 0.064 & 0.091 & -0.030 & 0.003 \\
\hline $\ln (\mathrm{d} 5 / \mathrm{d} 1)_{2005}-\ln (\mathrm{d} 5 / \mathrm{d} 1)_{1998}$ & 0.102 & 0.035 & 0.049 & 0.018 \\
\hline \multicolumn{5}{|l|}{ Frauen } \\
\hline $\ln (\mathrm{d} 9 / \mathrm{d} 1)_{2005}-\ln (\mathrm{d} 9 / \mathrm{d} 1)_{1998}$ & 0.078 & 0.010 & 0.015 & 0.053 \\
\hline $\ln (\mathrm{d} 9 / \mathrm{d} 5)_{2005}-\ln (\mathrm{d} 9 / \mathrm{d} 5)_{1998}$ & -0.002 & 0.028 & -0.048 & 0.018 \\
\hline $\ln (\mathrm{d} 5 / \mathrm{d} 1)_{2005}-\ln (\mathrm{d} 5 / \mathrm{d} 1)_{1998}$ & 0.080 & -0.018 & 0.062 & 0.035 \\
\hline
\end{tabular}

Die Zunahme der Lohnungleichheit ist in erster Linie auf ein Absinken der unteren Löhne gepaart mit dem gleichzeitigen $A n$ steigen der Löhne im mittleren und vor allem im oberen Bereich zurückzuführen. $\mathrm{Ob}$ und inwieweit dieses Phänomen durch Faktoren wie beispielsweise die Ausweitung von Teilzeitbeschäftigung (insbesondere bei Frauen, vgl. z. B. Bothfeld et al. 2005) oder eine sich verschärfende Lohndifferenzen nach beruflicher Klassenposition (Breen 1997) zu erklären ist, soll im nachfolgenden Abschnitt geprüft werden.

\subsection{ERGEBNISSE DER DEKOMPOSITION}

Mit Hilfe der von Juhn et al. (1993) vorgeschlagenen Methode zur Zerlegung von Ungleichheitstrends kann untersucht werden, inwieweit die im deskriptiven Teil diskutierten Veränderungen in den Ungleichheitsrelationen durch eine veränderte Wirkungsweise ungleichheitsgenerierender Mechanismen (wie z. B. der Zunahme von Lohndifferenzen zwischen Bildungsgruppen oder zwischen beruflichen Klassenpositionen), durch eine veränderte $\mathrm{Zu}$ sammensetzung der Gruppe der Erwerbstätigen (wie z. B. durch eine Expansion von Jobs im Dienstleistungssektor) und durch einen veränderten Einfluss unbeobachteter Einflussfaktoren erklärt werden kann ( $T a$ belle 2). ${ }^{12}$

Werden zunächst die Befunde für die männlichen Beschäftigten in den alten
Bundesländern betrachtet, so zeigt sich, dass der Anstieg der Gesamtungleichheit (Änderung im D9/D1-Verhältnis) um ca. $8 \%$ primär auf die Veränderungen in den Ungleichheitsmechanismen zurückgeführt werden kann. ${ }^{13}$ Dagegen wurde die Zunahme der Lohnungleichheit durch die Änderungen in der Zusammensetzung der Arbeitsmarktteilnehmer eher abgeschwächt (-0.041 log-Punkte). Oder anderes formuliert: Hätte sich die Zusammensetzung der Erwerbstätigen zwischen 1998 und 2005 nicht verändert, wäre der Zuwachs der Lohnungleichheit (D9/D1-Verhältnis) 2005 sogar um etwa vier Prozentpunkte höher ausgefallen. Schließlich zeigt sich, dass ein Teil des Anstiegs der Ungleichheit auf eine Veränderung der unbeobachteten Komponente zurückgeführt werden kann (0.034 log-Punkte). Der Blick auf die Änderungen der Ungleichheitsrelationen oberhalb bzw. unterhalb des Medianlohns offenbart ferner, dass in den beiden Bereichen der Verteilung teilweise unterschiedliche Prozesse wirksam waren. Erstens indu-

\footnotetext{
12 Für einen Vergleich mit Ergebnissen anderer Dekompositionsanalysen siehe Gernandt und Pfeiffer (2006) sowie Kohn (2006).

13 Für die Dekompositionsanalyse wurde der Bruttostundenlohn logarithmiert, sodass die Veränderungen in den Ungleichheitsrelationen durch die Transformation [exp(log-Punkte)-1]*100 als prozentuale Änderungen interpretiert werden können (z. B. D9/D1-Verhältnis: 0.082 log-Punkte = Veränderung um $8,55 \%$ ).
} 
zierte die veränderte Wirkungsweise ungleichheitsgenerierender Mechanismen in beiden Verteilungsbereichen eine Zunahme der Ungleichheit, wobei dieser Effekt für den unteren Bereich deutlich stärker ausfällt (0.024 vs 0.064 log-Punkte). Um diese Entwicklung sozialstrukturell möglichst differenziert zu verstehen, haben wir auch eine detaillierte Analyse einzelner Koeffizienten vorgenommen, die allerdings aus Platzgründen nicht tabellarisch dargestellt ist. Diese Analyse zeigt jedoch, dass die individuelle berufliche Klassenposition für beide Verteilungsbereiche mit Abstand den größten Einfluss auf die Änderung der Ungleichheitsrelationen hat. Insbesondere das unterdurchschnittliche Lohnwachstum bei un- bzw. angelernten Arbeitern wie bei Facharbeitern führte $\mathrm{zu}$ einer relativen Schlechterstellung dieser Gruppen gegenüber anderen Berufsklassen. So wuchs beispielsweise der relative Lohnabstand zwischen un- bzw. angelernten Arbeitern und Angestellten der oberen Dienstklasse um ca. 20 Prozentpunkte auf ca. $60 \%$. Dass vor allem diese Berufsklassen zu den Verlieren des post-industriellen Strukturwandels gehören, zeigen auch schon ältere Analysen, wie z. B. die von Esping-Andersen (1993) oder Goldthorpe (2002). Zweitens wird deutlich, dass die Veränderungen in der Zusammensetzung der Erwerbstätigen einen ungleichheitsreduzierenden Einfluss auf das D9/D5-Verhältnis ausübten, während sie im unteren Lohnbereich leicht ungleichheitserhöhend wirkten $(-0.055$ vs. 0.015 log-Punkte). Für dieses Muster gegenläufiger Ungleichheitstendenzen lassen sich nach unseren Analysen Veränderungen in der Branchenstruktur als ein plausibler Erklärungsfaktor ausmachen. Insbesondere der Einfluss des schrumpfenden Industrie- und des gleichzeitig wachsenden Dienstleistungssektors auf das Lohngefüge ist hierbei von Bedeutung. Dass solche Veränderungen innerhalb von Branchen lohnrelevant sein können, wird beispielsweise auch von Schettkat (2006) diskutiert.

Bei den Arbeitnehmerinnen in den alten Ländern fällt die Zunahme der Gesamtlohnungleichheit (D9/D1-Verhältnis: plus $13 \%$ ) deutlich stärker aus. Dafür sind vor allem Änderungen in der Wirkungsweise von Ungleichheitsmechanismen und weniger die Änderungen in der Zusammensetzung der Erwerbstätigen wichtig. Wird wiederum zwischen dem oberen und dem unteren Lohnbereich differenziert, zeigt sich, dass die Änderungen der Un- gleichheitsmechanismen sowohl im oberen als auch im unteren Bereich der Lohnverteilung wirksam waren ( 0.050 vs. 0.048$)$. In der detaillierten Analyse (nicht dargestellt) lässt sich erkennen, dass diesbezüglich vor allem Änderungen in den Lohnunterschieden zwischen den beruflichen Klassenpositionen einen verstärkenden Einfluss auf die Ungleichheit besitzen. ${ }^{14}$ Darüber hinaus erweist sich die zunehmende Lohnspreizung zwischen Groß- und Kleinbetrieben (das Lohndifferenzial wuchs um ca. 7 Prozentpunkte) als relevant, ein Ergebnis, das die Befunde von Gerlach und Hübler (1998) aus den 1990er Jahren bestätigt. Mit Blick auf die Veränderungen in der Zusammensetzung der Erwerbstätigen zeigt sich, dass diese für den oberen Lohnbereich leicht ungleichheitsreduzierend wirkten, während sie im unteren Lohnbereich einen ungleichheitserhöhenden Einfluss ausübten (-0.027 vs. 0.046 log-Punkte). Dieser gegenläufige Effekt ist vermutlich insbesondere auf den Abbau von Arbeitsplätzen im Industriesektor bei gleichzeitiger Ausweitung niedrig bezahlter Tätigkeiten im Dienstleistungssektor sowie im Bereich der Teilzeitarbeit zurückzuführen (Baethge/ Bartelheimer 2005; EU-Kommission 2006).

Für die männlichen Beschäftigten aus den neuen Bundesländern stieg die Lohnungleichheit zwischen 1998 und 2005 deutlich stärker an als für alle anderen Gruppen (D9/D1-Ratio: plus 17 \%). Dieses Ergebnis findet sich schon bei Kohn (2006) für den Zeitraum 1992-2001. Hauptquelle der wachsenden Ungleichheit ist die sich verändernde Wirkungsweise von ungleichheitsgenerierenden Mechanismen. Veränderungen in der Zusammensetzung der Erwerbstätigen sowie in den unbeobachteten Einflussfaktoren spielen dagegen eine nachgeordnete Rolle. Allerdings zeigen sich für den oberen und den unteren Lohnbereich unterschiedliche Zusammenhänge. Im oberen Lohnbereich sind vor allem die sich verändernden Lohndifferenziale zwischen Arbeitnehmergruppen wichtig (0.091 log-Punkte). Die zugrunde liegenden detaillierten Analysen zeigen, dass diesbezüglich vor allem die berufliche Klassenposition der Individuen entscheidend ist für die Position im ungleicher werdenden Lohngefüge der Bundesrepublik. Durch die Änderungen in der Zusammensetzung der Erwerbstätigen wurde die Zunahme der Lohnungleichheit dagegen eher abgeschwächt (-0.030 log-Punkte). Im Vergleich dazu stieg die Lohnungleichheit im unteren Verteilungsbereich sowohl aufgrund von Änderungen der ungleichheitsgenerierenden Mechanismen (insbesondere der Effekte der beruflichen Klassenposition) als auch aufgrund der veränderten Zusammensetzung der Erwerbstätigen (0.035 bzw. 0.049 log-Punkte). ${ }^{15}$

Bei Frauen in den neuen Bundesländern fällt der Anstieg der Gesamtungleichheit der Löhne in etwa so hoch aus wie bei den Männern in den alten Bundesländern. Die Veränderungen für die Gesamtverteilung sind vor allem durch Veränderungen in der unbeobachteten Komponente hervorgerufen und daher nicht durch die hier verwendeten Variablen zu erklären. Allerdings zeigt sich beim Blick auf den oberen und unteren Lohnbereich, dass Veränderungen in der Zusammensetzung der Erwerbstätigen ebenfalls einen entscheidenden Einfluss auf die Entwicklung der Lohnungleichheit ausübten (im oberen Bereich ungleichheitsreduzierend, im unteren Bereich ungleichheitserhöhend). Die empirischen Analysen legen nahe, dass dieser Entwicklung die Ausweitung von Dienstleistungstätigkeiten sowie von Teilzeitbeschäftigung bei gleichzeitigem Schrumpfen des Industriesektors wie auch des Bereichs des Öffentlichen Dienstes zugrunde liegt.

\section{Fazit}

In diesem Beitrag wurde auf der Grundlage von SOEP-Daten der Wandel von Ungleichheitsstrukturen in der Bundesrepublik zwischen 1998 und 2005 untersucht. Ausgangspunkt war die Beobachtung, dass

14 Auch hier ist die relative Verschlechterung der Löhne von un- bzw. angelernten Arbeiterinnen und Facharbeiterinnen besonders relevant. Allerdings fällt diese geringer aus als bei den Männern (z. B. erhöhte sich der Lohnabstand zwischen un- bzw. angelernten Arbeiterinnen und Angestellten der oberen Dienstklasse um 10 Prozentpunkte auf ca. $50 \%$ ).

15 Die Veränderungen in den Lohneffekten der beruflichen Klassenposition fallen für Ostdeutschland anders aus als für Westdeutschland. Hier verlieren alle Berufsklassen relativ gegenüber der oberen Dienstklasse, was unter anderem zu einer vergleichsweise starken Erhöhung der Lohnungleichheit im oberen Lohnbereich führt. Bezüglich der Veränderungen in der Zusammensetzung der Erwerbstätigen lassen sich (sowohl für den oberen als auch für den unteren Lohnbereich) keine eindeutigen Wirkungsfaktoren ausmachen. 
sich die Lohnungleichheit in der Bundesrepublik, nach einer Phase relativer Stabilität in den 1990er Jahren, wieder verstärkt hat. Mit der vorliegenden Analyse individueller Arbeitslöhne abhängig Beschäftigter wurde dabei hinterfragt, welchen Anteil eine veränderte Wirkungsweise ungleichheitsgenerierender Mechanismen (Änderung in den Lohndifferenzen zwischen bestimmten Gruppen von Arbeitnehmerinnen und Arbeitnehmern) bzw. eine veränderte $\mathrm{Zu}$ sammensetzung der Gruppe der Erwerbstätigen für die wachsende Lohnungleichheit in Deutschland hat.

Die empirischen Befunde zeigen zunächst, dass die Zeiten stabiler Ungleichheitsstrukturen in der Bundesrepublik der Vergangenheit angehören. Zusätzlich zur Umverteilung, wie sie sich in der wachsenden Kluft zwischen Einkommen aus abhängiger Beschäftigung und Unternehmensgewinnen zeigt (Statistisches Bundesamt 2007), hat auch innerhalb der Gruppe der abhängig Beschäftigten die Ungleichheit der Löhne im Zeitverlauf zugenommen. Die Lohndynamik zwischen 1998 und 2005 ist dabei durch ein Ansteigen der Löhne im mittleren und oberen Bereich bei gleichzeitigem Absinken unterer Lohnniveaus gekennzeichnet. Diese Entwicklung resultiert in einer Verstärkung der Ungleichheit der Löhne, die vor allem durch eine Zunahme der Ungleichheit im unteren
Bereich der Lohnverteilung gespeist wird. Ein vergleichender Blick auf die Lohnungleichheit in den neuen und alten Bundesländern ergibt, dass diese, bei bestehenden Besonderheiten, ähnlichen Dynamiken unterliegt.

In der Dekompositionsanalyse wurde daran anknüpfend gezeigt, dass in erster Linie die Veränderung ungleichheitsgenerierender Mechanismen für die steigende Gesamtlohnungleichheit verantwortlich ist. Hierbei erwies sich vor allem die Zunahme der Lohnabstände zwischen den Berufsklassen als dominant: Insbesondere die Löhne der un- oder angelernten Arbeiterinnen und Arbeiter, aber auch der Facharbeiterinnen und -arbeiter, haben sich im Vergleich zu den anderen Berufsklassen verschlechtert. Die Restrukturierung des deutschen Arbeitsmarktes der letzten Jahre trifft daher hauptsächlich die manuellen beruflichen Positionen und weniger die nicht-manuellen Berufsklassen. Man kann auf der Grundlage der Ergebnisse auch formulieren, dass die berufliche Position in Hinblick auf die Lohnverteilung gegenüber anderen Faktoren (z. B. Bildungsabschluss) relativ gesehen an Bedeutung gewinnt. $\mathrm{Zu}$ sätzlich können die Veränderungen in der Zusammensetzung der Erwerbstätigen für eine wachsende Ungleichheit der Löhne, vor allem im unteren Einkommensbereich, erklärend hinzugezogen werden. Hier sind es insbesondere die Verschiebungen in der Branchenstruktur und die Ausweitung von Teilzeitarbeit (Letzteres für Arbeitnehmerinnen), die zu einer Erhöhung der Lohnungleichheit am deutschen Arbeitsmarkt beigetragen haben.

Abschließend kann die These formuliert werden, dass es die Befunde dieses Beitrags nicht mehr plausibel erscheinen lassen, von einer Nivellierung sozialer Ungleichheit in der Bundesrepublik zu sprechen. Vielmehr legen die empirischen Analysen nahe, dass sich die Lohnungleichheit nach einer Phase relativer Stabilität wieder erhöht hat. Daher scheint es für die Zukunft angebracht, mit anknüpfenden Analysen eine potenziell weiter zunehmende Ungleichheit der Einkommen und Löhne näher zu untersuchen. So wäre es beispielsweise interessant, über längere Zeiträume zu prüfen, welche Folgen die Strukturreformen der letzten Jahre (Stichworte: Hartz IV, Absenkung des Spitzensteuersatzes) oder der ökonomische Aufschwung der Jahre 2006 und 2007 für die Entwicklung der Lohn- und Einkommensverteilung haben. Ferner scheint es lohnend, nicht nur Kernbeschäftigte des Arbeitsmarktes in einer solchen Analyse zu berücksichtigen, sondern auch Randgruppen des Arbeitsmarktes oder Selbstständige mit einzubeziehen. 
Atkinson, A. B. (2000): The Changing Distribution of Income: Evidence and Explanations, in: German Economic Review 1, S. 3-18

Baethge, M./ Bartelheimer, P. (2005): Deutschland im Umbruch. Ergebnisse und Perspektiven des sozioökonomischen Berichtsansatzes; in: SOFI-Mitteilungen 33, S. 17-29

Berger, J. (2005): Nimmt die Einkommensungleichheit weltweit zu?, in: Leviathan 4, S. 464-481

Bergmann, J. (2004): Die Reichen werden reicher - auch in Deutschland; in: Leviathan 2, S. 185-202

Bothfeld, S./Klammer, U./Klenner, C./Leiber, S./Thiel, A./ Ziegler, A. (2005): WSI-FrauenDatenReport 2005. Handbuch zur wirtschaftlichen und sozialen Situation von Frauen, Berlin

Breen, R. (1997): Risk, Recommodification and Stratification; in: Sociology 3, S. 473-489

Deutsche Schutzvereinigung für Wertpapierbesitz (DSW) (2006a): DSWDividendenuntersuchung, URL: http://www.dsw-info.de

Deutsche Schutzvereinigung für Wertpapierbesitz (DSW) (2006b): DSW-

Studie Vorstandsvergütung, URL: http://www.dsw-info.de

Erikson, R./Goldthorpe, J. H. (1992): The Constant Flux, Oxford

Esping-Andersen, G. (1993): Post-industrial class structures. An analytical framework, in: Esping-Andersen, G. (Hrsg.): Changing Classes. Stratification and Mobility in Post-industrial Societies, London, S. 7-31

EU-Kommission (2006): Employment in Europe, Luxemburg

Gerlach, K./Hübler, O. (1998): Firm size and wages in Germany - trends and impacts of mobility; in: Empirica 3, S. 245-261

Gernandt, J./Pfeiffer, F. (2006): Rising Wage Inequality in Germany, ZEW Discussion Paper 06-019, Mannheim

Goldthorpe, J. H. (2002): Globalisation and Social Class; in: West European Politics 3, S. 1-28

Hinz, T./Abraham, M. (2005): Theorien des Arbeitsmarkts: Ein Überblick, in: Hinz, T./Abraham, M. (Hrsg.): Arbeitsmarktsoziologie, Wiesbaden, S. $17-68$

Holst, E. (2003): Einkommensunterschiede zwischen Frauen und Männern in höheren Positionen; in: WSI-Mitteilungen 4, S. 243-250

Hradil, S. (2005): Warum werden die meisten entwickelten Gesellschaften wieder ungleicher? in: Windolf, P. (Hrsg.): Finanzmarkt-Kapitalismus. Analysen zum Wandel von Produktionsregimen, Kölner Zeitschrift für Soziologie und Sozialpsychologie, Sonderheft 45, Wiesbaden, S. 460-483
Juhn, C./Murphy, K. M./Pierce, B. (1993): Wage Inequality and the Rise in Returns to Skill; in: The Journal of Political Economy 3, S. 410-442 Katz, L. F./Autor, D. H. (1999): Changes in the Wage Structure and Earnings Inequality, in: Ashenfelter, O./Card, D. (Hrsg.): Handbook of Labor Economics, Amsterdam, S. 1463-1555

Kohn, C. (2006): Rising Wage Dispersion, After All! The German Wage Structure at the Turn of the Century, IZA Discussion Paper 2098, Bonn Liebeskind, U. (2004): Arbeitsmarktsegregation und Einkommen. Vom Wert "weiblicher" Arbeit; in: Kölner Zeitschrift für Soziologie und Sozialpsychologie 4, S. 630-653

Möller, J. (2005): Die Entwicklung der Lohnspreizung in West- und Ostdeutschland, in: Bellmann, L./Hubner, O./Meyer, W./Stephan, G. (Hrsg.): Institutionen, Löhne und Beschäftigung, Nürnberg, S. 47-63

Prasad, E. S. (2004): The Unbearable Stability of the German Wage Structure: Evidence and Interpretation; in: IMF Staff Papers 2, S. 354-385 Schäfer, C. (2006): Unverdrossene "Lebenslügen-Politik" - Zur Entwicklung der Einkommensverteilung; in: WSI Mitteilungen 11, S. 583-591 Schettkat, R. (2006): Lohnspreizung: Mythen und Fakten, Düsseldorf Schulten, T. (2006): Europäischer Tarifbericht des WSI - 2005/2006, in: WSI-Mitteilungen 7, S. 365-373 SOEP Group (2001): The German Socio-Economic Panel (GSOEP) after more than 15 years - Overview. Proceedings of the 2000 Fourth International Conference of German Socio-Economic Panel Study Users (GSOEP2000); in: Vierteljahreshefte zur Wirtschaftsforschung 1, S. 7-14 Statistisches Bundesamt (2007): Pressemitteilung vom 11.01., URL: http://www.destatis.de/presse Steiner, V./Hölzle, T. (2000): The Development of Wages in Germany in the 1990's - Description and Explanations, in: Hauser, R./Becker, I. (Hrsg.): The Personal Distribution of Income in an International Perspective, Berlin, S. 7-30

Weins, C. (2005): Die Entwicklung der Lohnungleichheit in Deutschland und den USA zwischen 1980 und 2000, in: Windolf, P. (Hrsg.): Finanzmarkt-Kapitalismus. Analysen zum Wandel von Produktionsregimen. Kölner Zeitschrift für Soziologie und Sozialpsychologie, Sonderheft 45, Wiesbaden, S. 484-503 